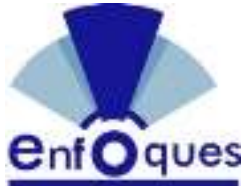

\section{GESTIÓN LOGÍSTICA EN LAS INSTITUCIONES UNIVERSITARIAS PÚBLICAS DE LA COSTA ORIENTAL DEL LAGO}

\author{
LOGISTIC MANAGEMENT IN THE PUBLIC UNIVERSITY \\ INSTITUTIONS OF THE EAST COAST OF THE LAKE
}

ROSSANY GARCÍA

Artículo recibido diciembre 2019 | Arbitrado enero 2020 | Publicado 01 de abril 2020

\begin{abstract}
Resumen
La presente investigación tuvo como objetivo general analizar la gestión logística en la unidad de compra de las instituciones universitarias públicas de la Costa Oriental del Lago. Metodológicamente se tipifico como una investigación descriptiva, con un diseño no experimental, transeccional y de campo. La población quedo constituida por las instituciones universitarias públicas de la Costa Oriental del Lago, siendo las unidades informantes veinticuatro (24) sujetos entrecoordinadores, jefes de compras, compradores y personal de apoyo. La recolección de los datos se realizó a través de un cuestionario dicotómico compuesto por 33 ítems, los cuales fueron validados por 5 expertos. Para determinar la confiabilidad se utilizó el método de Küder - Richardson, obteniéndose una confiabilidad de 0,90. La información fue procesada a través de la estadística descriptiva mediante la frecuencia relativa. Se evidencio moderado cumplimiento de la gestión logística, permitiendosatisfacerparcialmente las necesidades de las dependencias académicas administrativas cuando requieren materiales.
\end{abstract}

Palabras clave: Cumplimiento, gestión logística, materiales, necesidades, unidad de compra

\section{Abstract}

The purpose of this research was to analyze the logistics management in the purchasing unit of the public university institutions of the East Coast of the Lake. Methodologically it was typified as andescriptive investigation, with a non-experimental, transectional and field design. The population was constituted by the public university institutions of the East Coast of the Lake, the twenty-four (24) reporting units being subjects among coordinators, purchasing managers, buyers and support staff. Data collection was carried out through a dichotomous questionnaire composed of 33 items, which were validated by 5 experts. To determine the reliability, the Küder - Richardson method was used, obtaining a reliability of 0.90 . The information was processed through descriptive statistics using relative frequency. Moderate compliance with logistics management was evidenced, allowing partially meet the needs of administrative academic units when they require materials.
\end{abstract}

Key words: Compliance, logistics management, materials, needs, purchasing unit

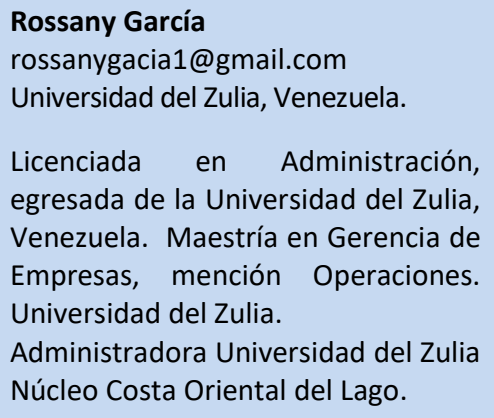




\section{INTRODUCCIÓN}

La nueva realidad competitiva presenta un campo de batalla en donde la flexibilidad, la velocidad de llegada al mercado y la productividad serán las variables claves que determinarán la permanencia de las empresas en los mercados. De esta manera, una de las herramientas esenciales para abordar el plan estratégico de gestión de una empresa, consiste en aplicar un sistema de gestión logística, partiendo de la evaluación de la empresa a través de sus factores internos y externos a fin de reforzar sus ventajas competitivas.

En este orden de ideas, para Reyes (2001), el concepto con el cual se introdujo a la logística se enfocaba al manejo de todas las actividades que facilitan el movimiento de productos, la coordinación de la oferta y la demanda, la optimización de la utilidad en el tiempo y la producción, para ofrecer el producto esperado, en el lugar preciso, con la cantidad requerida, en el tiempo justo a un costo adecuado.

De esta forma, todas aquellas actividades donde se involucre el movimiento de materias primas, materiales y otros insumos forman parte de los procesos logísticos, al igual de aquellas tareas que ofrecen un soporte adecuado para la transformación de dichos elementos en productos terminados: las compras, el almacenamiento, la administración de los inventarios, el mantenimiento de las instalaciones y maquinarias, la seguridad y los servicios de planta (suministros de agua, gas, electricidad, combustibles, aire comprimido, vapor, entre otros).

No obstante, para la asociación Council of Logistics Management (2001) la definición más concreta de este término es:

El proceso de planear, implantar y controlar de manera eficiente

\begin{abstract}
y económica el flujo y almacenamiento de materias primas, inventarios en proceso, productos terminados $\mathrm{e}$ información vinculada con ellos desde el punto de origen al punto de consumo con el propósito de adecuarse a los requerimientos del cliente. ( $p$. 32)
\end{abstract}

En otras palabras, se entiende por logística al conjunto de conocimientos, acciones y medios destinados tanto a prever como proveer los recursos necesarios para realizar una actividad principal en tiempo idóneo, forma adecuada, al menor costo, en un marco de productividad y calidad. En este contexto, la logística se ha convertido en una herramienta básica para resolver la presión competitiva y para propiciar el desarrollo estratégico e integral de las empresas ante los cambios del mundo.

Del mismo modo, González (2005) señala que la logística da como ventaja la coordinación con los proveedores, la mejora de rotación de los inventarios, un servicio o producción más seguro, ahorros en embalaje y manipulación de inventarios, la mejor coordinación, evita duplicidad de esfuerzos y la centralización de la responsabilidad. Bajo esta perspectiva, la logística permite, proporcionar un flujo de materiales, suministros necesarios para el buen funcionamiento de la organización y reduciendo las pérdidas a un nivel mínimo, estableciendo normas de calidad adecuadas, proveedores competentes, adecuados sistemas de almacenaje para la preservación de los insumos, sobre todo los perecederos, además de los servicios necesarios al precio más bajo posible.

Se infiere entonces, que la logística busca gerenciar estratégicamente la adquisición, el movimiento, el 
almacenamiento de productos y el control de inventarios, así como todo el flujo de información asociado, a través de los cuales la organización y su canal de distribución se encauzan de modo tal, que la rentabilidad presente y futura de la empresa sea maximizada en términos de costos $y$ efectividad. Esto indica, que la logística desde el punto de vista gerencial es una estrategia necesaria para manejar de forma integral la cadena de suministros, de tal forma que logre el balance óptimo entre las necesidades del cliente y los recursos disponibles de la empresa y su desempeño debe ser medido a través del servicio al cliente final.

Por otra parte, para medir con efectividad el desempeño de la empresa, es necesario describir cada uno de los elementos del sistema de gestión logística, valorando y dimensionando los mismos mediante la evaluación de la cadena de valor, la cual categoriza las actividades que generan valor agregado en la organización. En este sentido, el análisis de la cadena de valor comienza con el reconocimiento de las actividades que se llevan a cabo para diseñar, producir, comercializar, entregar y apoyar su producto. Por lo tanto, las actividades logísticas deben coordinarse entre sí para lograr mayor eficiencia en todo el sistema productivo. Por dicha razón, la logística no debe verse como una función aislada, sino como un proceso global de generación de valor para el cliente, esto es, un proceso integrado de tareas que ofrezca una mayor velocidad de respuesta al mercado, con costos mínimos.

Al respecto, señala Porter (2001), que las empresas desarrollan su ventaja competitiva, sobre su tipo de actividades, basándose las mismas en el costo; es decir, se deben estudiar los canales de distribución del producto hasta su destinatario final. De esta manera, el canal de distribución de cualquier producto $\mathrm{y} / \mathrm{o}$ actividad es el camino seguido por el producto en su movimiento desde el fabricante hasta el consumidor final, es decir son los canales a través de los cuales se llevan a cabo las ventas y se distribuyen los productos.

Ante esta realidad, están inmersas las instituciones universitarias públicas de la Costa Oriental del Lago, las cuales realizan esfuerzos para mejorar continuamente en la calidad de su servicio y revisiones constantes de sus procesos, con la intención de desarrollar ventajas competitivas. Específicamente en la Costa Oriental del Lago existen instituciones universitarias públicas que deben competir para conservar un nivel adecuado a fin de ofrecer sus servicios como alternativas de estudios para los individuos quienes desean obtener un grado universitario en este tipo de instituciones.

En este sentido, se entiende que con la velocidad de aparición de nuevas herramientas, dispositivos, sistemas así como sus aplicaciones relacionados con las tecnologías de información que garanticen el proceso de compra de los insumos; además de los materiales adquiridos para todas las dependenciaspermitiendo con ello el desarrollo de modelos académicos con productividad y calidad, se hace notablemente complejo, para las universidades, mantenerse a la vanguardia de la incorporación de éstas y su aprovechamiento académico, dado el dinamismo del mercado.

Por lo tanto, es vital para el proveedor conocer a fondo el comportamiento del consumidor de las instituciones de educación superior, y los factores que influyen en el proceso de decisión de compra, pues, debido a su carácter de compradores institucionales, poseen características propias a su 
naturaleza haciéndolos así, enocasiones atractivos para los proveedores, destacándose por el tamaño de sus pedidos, menor frecuencia de compra, lo cual, trae como consecuencia que cada venta sea de vital importancia para las organizaciones vinculadas en el proceso de compra-venta.

Es por ello, que el desconocimiento del comportamiento del comprador, puede traer ciertas consecuencias negativas para las empresas proveedoras tales como; errores en detectar tendencias de compra, anticipaciones al mercado desacertadas o fuera del contexto, clientes insatisfechos, disminución de la competitividad, perdida de posicionamiento, desmejoramiento de la imagen corporativa, reducción del ciclo de vida organizacional, es decir, de su permanencia en el tiempo y estrategias de marketing desenfocadas o inadecuadas.

Aunado a esta realidad, las instituciones universitarias públicas para llevar a cabo su proceso de compra, están reguladas por normas y procedimientos para la adquisición de bienes y contratación de servicios y obras, elaboradas por sus respectivas comisiones de contrataciones de la unidad de compra en concordancia con la normativa legal vigente, según decreto Nro. 5.929 con Rango, Valor y Fuerza de la Ley de Contrataciones Públicas, publicado en Gaceta Oficial de la República Bolivariana de Venezuela Nro. 360.129 Extraordinario de fecha 25 de marzo de 2008 (DRVFLCP).

Igualmente, según decreto 2.371 publicado en Gaceta Oficial de la República Bolivariana de Venezuela Nro. 37.688 de fecha 13 de mayo de 2003 donde se reglamenta los procedimientos de adjudicación directa, el decreto 4.998 publicado en Gaceta Oficial de la República Bolivariana de Venezuela Nro. 38.567 de fecha 20 de noviembre de 2006, mediante el cual se dictan las "Medidas temporales para la Promoción y Desarrollo de las Pequeñas y Medianas Industrias, Cooperativas y otras formas asociativas, productivas de bienes, prestadoras de servicios y ejecutoras de obras ubicadas en el país".

Asimismo, el decreto Nro. 3.776 mediante el cual se dicta el reglamento 1 de la Ley Orgánica de Administración Financiera del Sector Público, sobre el Sistema Presupuestario, que data de julio de 2005, publicado en Gaceta Oficial Extraordinaria de la República Bolivariana de Venezuela Nro. 5.781, donde se reglamentan los procedimientos para el manejo de los fondos de avance y los fondos de anticipo de los órganos de la República. Asimismo, se ajusta a la Ley de Universidades y Ley contra la corrupción publicada en Gaceta Oficial Nro. 5.637 de fecha 07 de abril de 2003.

Ahora bien, a través de la observación directa efectuada en este sector, además de algunas entrevistas realizadas al personal involucrado en el área objeto de estudio, evidencian la falta de un sistema de logística más eficiente y eficaz dentro de sus procesos, ya que estos deben cubrir varias etapas desde el momento de la solicitud por parte de quien tenga la necesidad de la compra hasta el proceso de la compra misma. Dicha necesidad en primer lugar se debe ajustar a lo que se tiene en el plan de compra y presupuesto anual; posterior a ello, se prepara una invitación a tres empresas, para evaluar las condiciones de la compra, según este análisis la comisión emite una carta de recomendación que es enviada a la máxima autoridad para que éste emita una carta de adjudicación y poder realizar la compra.

Dicho proceso puede durar igual o mayor a 10 días, lo que se traduce en retraso en las actividades tanto administrativas como operativas de las instancias que hizo el requerimiento. Es por ello, que surge la 
necesidad y el interés de analizar la gestión de logística en la unidad de compra de las instituciones universitarias públicas de la Costa Oriental del Lago. A fin de aportar soluciones logísticas viables a las instituciones objeto de estudio, para mejorar la eficiencia de las mismas, además de establecer elementos teóricos que ayuden a implantar además de mejorar el servicio que se presta y al mismo tiempo, dicha información contribuya a nivel gerencial en la toma de decisiones para realizar las correcciones necesarias con un soporte debidamente documentado.

Con base al planteamiento realizado, se considera formular el problema de la siguiente manera: ¿Cómo es la gestión logística en la unidad de compra de las instituciones universitarias públicas de la Costa Oriental del Lago?

\section{Gestión logística}

Ivancevich y otros (2000) refieren que el termino gestión es "el proceso emprendido por una o más personas para coordinar las actividades laborales de otras personas con la finalidad de lograr resultados de alta calidad que cualquier otra persona, trabajando sola no podría alcanzar". Según estos autores, para que pueda haber gestión, debe estar un equipo de personas que coordinen todas las actividades laborales y de esta manera logren calidad en los procesos/productos, lo cual no se lograría trabajando de manera individual, lo importante es el trabajo en equipo.

En este sentido, Koontz y Weihrich (2003), plantean que el proceso comprende la administración de unos recursos y medios de una organización para lograr desarrollarlo y obtener un producto que permita cumplir con los objetivos y metas de la organización.

Las anteriores definiciones de gestión, presentan elementos como, personas, conocimiento, actividades, productos, medios, recursos, entre otros. Asimismo, el concepto de gestión está relacionado con el concepto de administración y de gerencia, lo esencial es que este concepto hace referencia a las acciones de planificar, organizar y controlar que se vinculan entre sí mediante la función de liderar.

Asimismo, la planificación determina los objetivos, qué resultados ha de lograr la empresa; pues de esta manera, la organización especifica las acciones con las cuales se lograran los resultados planificados, el control realiza un seguimiento a las acciones para obtener los resultados y objetivos previstos; todos estos dirigidos, motivados por la función de liderar. En otras palabras, gestión representa las acciones plasmadas en el plan estratégico y tecnológico de una organización con la finalidad única de lograr el éxito en el alcance de los objetivos empresariales, involucrando para ello el personal que en ella labora.

Desde esta perspectiva, se considera la logística como un elemento integrador del éxito de las organizaciones. En este sentido, el término "logística" (del inglés: Logistics) ha sido tomado del ámbito militar para ser utilizado en el mundo empresarial como el término que, en un sentido general, se refiere: a) al posible flujo de los recursos que una empresa va a necesitar para la realización de sus actividades; y b) al conjunto de operaciones y tareas relacionadas con el envío de productos terminados al punto de consumo o de uso. Por tanto, no es una exageración el decir que el éxito final de un proyecto depende en una buena parte, de la logística.

Por ello, es imprescindible que el mercadólogo tenga un buen conocimiento de este importante tema; por lo cual, en la presente investigación se introduce en lo 
que es la logística, brindándole la respuesta de una pregunta básica pero fundamental: ¿cuál es la definición de logística?

Según Ferrer y otros (2004) la logística es una función operativa importante que comprende todas las actividades necesarias para la obtención y administración de materias primas y componentes, así como el manejo de los productos terminados, su empaque y su distribución a los clientes". Introducción a los Negocios en un Mundo Cambiante.

De igual manera, para Ballou (2004), es todo movimiento y almacenamiento que facilite el flujo de productos desde el punto de compra de los materiales hasta el punto de consumo, así como los flujos de información que se ponen en marcha, con el fin de dar al consumidor el nivel de servicio adecuado a un costo razonable"

Por su parte Cespón y Auxiliadora (2003) la definen como el proceso de gestionar los flujos material e informativo de materias primas, inventario en proceso, productos acabados, servicios y residuales desde el suministrador hasta el cliente, transitando por las etapas de gestión de los aprovisionamientos, producción, distribución física y de los residuales".

En ese orden de ideas, para Lamb y otros (2002), consideran la logística, como el proceso de administrar estratégicamente el flujo y almacenamiento eficiente de las materias primas, de las existencias en proceso y de los bienes terminados del punto de origen al de consumo.

$Y$ finalmente Anaya (2000), la define como el control del flujo de materiales desde la fuente de aprovisionamiento hasta situar el producto en el punto de venta de acuerdo con los requerimientos del cliente.

En síntesis, se puede adoptar la siguiente definición de logística para conocer y describir de una forma amplia y precisa lo que es la logística en el contexto empresarial: La logística es una función operativa que comprende todas las actividades y procesos necesarios para la administración estratégica del flujo y almacenamiento de materias primas $y$ componentes, existencias en proceso y productos terminados; de tal manera, que éstos estén en la cantidad adecuada, en el lugar correcto y en el momento apropiado.

De los planteamientos anteriores, se enmarca la logística como un proceso gerencial, en el cual según Pau i Cos y Navascues (2000), es vista como una herramienta de "Management" total y conduce a dirigir todos los cambios tanto estructurales como culturales de las empresas para incrementar la competitividad y rentabilidad. La gestión logística agrupa las actividades que ordenan los flujos de materiales, coordinando recursos y demandas para asegurar un nivel determinado de servicio al menor costo posible, es decir, proporciona una total visibilidad del proceso en el cual se gestiona.

En este sentido para Ballou (2004), la gestión logística es la gobernanza de las funciones de la cadena de suministro que incluyen la gestión de transporte interno y externo, la gestión de flotas, el almacenamiento, la manipulación de materiales, el cumplimiento de órdenes, el diseño de redes logísticas, la gestión de inventario, la planificación de oferta/demanda y la gestión de proveedores de logística externos.

En el mismo ámbito el autor plantea que para llevar a cabo la gestión logística se deben establecer las funciones de logística como lo son el servicio al cliente, el suministro y adquisición, la planificación de la producción y el embalaje y ensamblaje. La gestión de logística es parte de todos los niveles de planificación y ejecución - 
estratégica, operativa y táctica. Es una función integradora, que coordina todas las actividades logísticas, y también integra actividades logísticas con otras funciones, incluyendo la comercialización, las ventas de producción, las finanzas y la tecnología de la información.

No es por lo tanto una actividad funcional sino un modelo, un marco referencial; no es una función operacional, sino un mecanismo de planificación, la logística es una manera de pensar que permitirá incluso reducir la incertidumbre en un futuro desconocido. Es entonces un conjunto de medios y métodos necesarios para llevar a cabo la organización de una empresa o de un servicio, especialmente de distribución.

En el mismo orden de ideas, para López (2001), la gestión logística se constituye en el componente principal de la cadena de valor que incorpora el producto. La logística vela por la optimización y el mantenimiento de los recursos de esta cadena a través de sistema de información compartidos por todos los que intervienen en ella y mediante la aplicación de indicadores de desempeño que permitan conocer los niveles de inventarios, los tiempos de procesamiento, la rotación de los productos en los supermercados, entre otros.

Mientras que, Schroeder (2004), describe la gestión logística como el medio por el cual se satisfacen las necesidades de los clientes a través de la materia prima y sus operaciones a más de esta hasta los proveedores. La misión de la gestión logística es planificar y coordinar todas las actividades necesarias para conseguir los niveles deseados de servicio y calidad al menor costo posible. En consecuencia, la logística debe contemplarse como el enlace entre el mercado y la actividad operativa de la empresa.
En síntesis, la gestión logística es primordial para casi todas las áreas de las organizaciones, pero es solo en estos últimos años que las empresas han comenzado a tratar de implementar a gran escala las actividades logística, para así resolver problemas relacionados a transporte, distribución y almacenamiento de productos por parte de la empresa, que siempre han sido un caso difícil de atender.

\section{Actividades claves de gestión de logística}

Las actividades claves son primordiales para las funciones logísticas ya que afectan más al costo total de la cadena de suministro. Entre estas actividades se distinguen: servicio al cliente, transporte, gestión de inventarios y flujo de información y procesamiento de pedidos. Estas suelen variar de una empresa a otra, dependiendo de su estructura organizacional, desarrollo de operaciones o del grado de madurez en la implantación del concepto de logística integral.

Ahora bien, Montero (2000), plantea las actividades logística deben coordinarse entre sí para lograr mayor eficiencia y eficacia en todo el sistema productivo. Por dicha razón, la logística no debe verse como una función aislada, sino como un proceso global de generación de valor para el cliente, esto es integrando tareas que ofrezcan una mayor velocidad de respuesta al mercado, con costo mínimo. En dicho proceso se debe abarcar: distribución, compra, recepción, almacenes, inventarios, fabricación, expedición.

En efecto, la gestión logística debe desarrollarse con la finalidad de asegurar que los procesos de la organización se planifiquen y desarrollen contemplando los requisitos logísticos del cliente. Los procesos 
logísticos deberían considerarse como procesos específicos de la organización cuyo objetivo es apoyar la calidad de los productos elaborados por la organización, así como gestionar el riesgo de no cumplir con los requisitos logísticos del cliente.

De acuerdo a esto Mora (2006), aporta que las empresas tienen grandes vacíos en la medición del desempeño de las actividades logística, razón por la cual es importante establecer indicadores de gestión logística para evitar barreras a la alta gerencia, en la identificación de los principales problemas presentes en la cadena logística, que perjudican ostensiblemente la competitividades de las empresas.

Por su parte, Ballou (2004) divide las actividades de la logística en dos tipos: las claves y las de apoyo. Este autor explica que la diferencia entre las actividades clave y de apoyo se hace debido a que algunas actividades siempre van a tener lugar en cualquier canal logístico, mientras que las otras solo se desarrollan bajo ciertas circunstancias y características del proceso de la empresa.

Por su parte, Garrioz (2005) plantea que las actividades vinculadas a la gestión de logística a nivel estratégico envuelve a todas las áreas de la compañía para diseñar, desde las etapas de identificación de la necesidad y concepción de los productos y/o servicios, un proceso que incluya todos los medios necesarios para obtener los mejores resultados, en términos económicos y de satisfacción del consumidor.

Ampliando esta información, Stock y Kasarda (1999), señala que la gestión logística es una función integrada que coordina y optimiza todas las actividades claves, así mismo las integra con otras funciones incluyendo marketing, ventas, manufactura, finanzas y las tecnologías de la información.
En este sentido, para los autores la gestión logística desempeña un papel de coordinación que puede conceder a la empresa una ventaja competitiva; por tanto, las actividades de gestión que incluye son diversas, desde el servicio al cliente, transporte, entrada, salida y transformación de materias primas, almacenamiento, manejo de inventarios manipulación de materiales y/o productos, realización de pedidos, diseño de la red logística, gestión de inmovilizado, planificación de pedidos (suministrados y demandas) y las relaciones con proveedores de servicios logísticos.

La logística así entendida tiene un carácter multifuncional: alcanzar el objetivo final de la forma más eficiente posible no depende solo de una función o responsabilidad de la empresa, sino que es el resultado de aunar los esfuerzos de toda organización. De ahí la necesidad de contemplar todo el proceso logístico desde la perspectiva estratégica propia de la alta dirección de la empresa.

Para los efectos investigativos se abordan las actividades claves: planificación logística, servicio al cliente, transporte, manejo de inventario, flujo de información de pedido tomando en consideración que en las instituciones universitarias estas son las que se manejan al momento de gestionar la logística en las unidades de compras.

\section{Actividades de apoyo}

Una vez identificado las actividades claves que conforman el proceso logístico, resta definir las actividades de apoyo, las cuales se agruparan en procesos denominados de apoyo, y facilitan el desarrollo de las actividades integrantes de los procesos antes mencionados.

Ogalla (2005), señala que en estos procesos de apoyo o soporte, se identifican aquellas actividades, que si bien, no son 
parte de la actividad principal de la organización, son necesarias e imprescindibles para la buena gestión y desarrollo de la misma. De igual manera este conjunto de actividades aportan recursos al proceso de negocio.

En este sentido Mañueco (2004) añade que para su respectiva identificación se debe conocer: a) ¿Cuáles son los recursos necesarios para llevar a cabo los procesos clave? Y b) ¿Cómo se garantiza la adecuada disposición y gestión de esos recursos?

En este orden de ideas, Pérez (2009) define los procesos de apoyo, como aquellos procesos que proporcionan las personas $y$ los recursos físicos necesarios por el resto de procesos y conforme con los requisitos de sus clientes internos.

Bajo este enfoque, pero abordando el proceso logístico la investigadora considera como actividades de apoyo: Almacenamiento, manejo de materiales, compras, cooperaciones con producción y el mantenimiento de información.

\section{Elementos de la gestión logística}

Acevedo y otros (2001), expresan que en la gestión del sistema logístico empresarial se identifican una serie de elementos o partes componentes del subsistema de carácter material, y que estos elementos son (aprovisionamiento, producción, distribución física y abastecimiento)

Al respecto, Taboada (1999), refiere que si se toma como referencia el flujo material, que es la columna vertebral de la logística, pueden entonces identificarse los elementos del sistema de gestión de logística los cuales son (aprovisionamiento, producción, distribución física y abastecimiento).
Como puede notarse, en ambos autores, existe coincidencia en las posiciones de los elementos que conforman la gestión de logística, los cuales forman parte del proceso logístico (aprovisionamiento, producción/operaciones, distribución física y abastecimiento).

Las bases teóricas que se abordaron, permitieron sustentar la variable gestión logística e incluir una definición desde la perspectiva de la investigadora como el conjunto de elementos destinados a prever y proveer los recursos necesarios que posibiliten realizar las actividades principales y de apoyo en tiempo, forma y al costo más oportuno para dirigir todos los cambios tanto estructurales como culturales de las instituciones universitarias públicas de la Costa Oriental del Lago para incrementar la competitividad y rentabilidad.

\section{MÉTODO}

Para el desarrollo de este estudio se realizó un abordaje no experimental de tipo descriptivo bajo el diseño de campo transeccional. La población quedó conformada por las instituciones universitarias públicas de la Costa Oriental del Lago, entre las cuales se encuentran: Universidad del Zulia en el Núcleo Costa Oriental del Lago, Universidad Nacional Experimental "Rafael María Baralt, y el Instituto Universitario de Tecnología Cabimas ubicadas en Venezuela.

Las unidades de observación o unidades informantes de cada una de las instituciones de educación superior, fueron los coordinadores, jefes de compras, comprador, personal de apoyo, los cuales se especifican en el tabla 1. 
Tabla 1. Población de estudio

\begin{tabular}{lccccc}
\hline $\begin{array}{l}\text { Instituciones } \\
\text { Universitarias }\end{array}$ & Coordinadores & $\begin{array}{c}\text { Jefe de } \\
\text { compras }\end{array}$ & Comprador & $\begin{array}{c}\text { Personal de } \\
\text { apoyo }\end{array}$ & Total \\
\hline $\begin{array}{l}\text { Universidad del } \\
\text { Zulia Núcleo COL }\end{array}$ & 2 & 1 & 1 & 2 & 6 \\
UNERMB & 2 & 1 & 2 & 4 & 9 \\
IUTC & 1 & 1 & 2 & 5 & 9 \\
\hline Total & $\mathbf{5}$ & $\mathbf{3}$ & $\mathbf{5}$ & $\mathbf{1 1}$ & $\mathbf{2 4}$ \\
\hline
\end{tabular}

Latécnica de recolección de datos empleada fue la encuesta integrada por un cuestionario como instrumento de medición, de frecuencia, escrito y auto administrado.EI cuestionario de la investigación, fue diseñado con un conjunto de preguntas cerradas, conformado por 33 ítems con dos (2) alternativas de respuestas correspondiente a una escala tipo Dicotómica $1=\mathrm{SI}$ y $0=\mathrm{NO}$.

Con el fin de validar el instrumento diseñado, este fue sometido al juicio de cinco (05) expertos para su revisión y su consecuente validación, compuesto por cinco (05) expertos tres (03) en el área de contenido y dos (02) metodológicos.Para calcular la confiabilidad del instrumento de recolección de datos, se utilizó el método denominado Coeficiente de KüderRichardson, obteniéndose una muy alta confiabilidad igual a 0,90.

Una vez obtenidos los resultados se organizaron agrupándolos por dimensión y así poder efectuar un análisis de la información, a través del baremo de interpretación mostrado en la tabla 2 .

Tabla 2. Baremo de interpretación

\begin{tabular}{ccc}
\hline Alternativa de respuesta & Intervalo & Categoría \\
\hline $\mathrm{Si}$ & $75 \% \leq X<100 \%$ & Alta aplicación \\
$\mathrm{Si}$ & $50 \% \leq X<75 \%$ & Moderada aplicación \\
$\mathrm{Si}$ & $25 \% \leq X<50 \%$ & Baja aplicación \\
$\mathrm{Si}$ & $0 \% \leq X<25 \%$ & Muy Baja aplicación \\
\hline
\end{tabular}

\section{RESULTADOS}

En la tabla 3, se presenta los datos alcanzados para la dimensión actividades claves, reflejando, de acuerdo a los encuestados, una concentración de respuestas afirmativas del $46,25 \%$, indicando que se da baja presencia a las actividades claves de la gestión logística presentes en la unidad de compra de las instituciones universitarias públicas de la Costa Oriental del Lago.

Al ver los resultados se identifican con alta presencia la planificación logística, moderada presencia el servicio al cliente, con baja presencia el transporte así como el flujo de información de pedido y con muy baja presencia el manejo de inventario. A juicio de la investigadora estos aspectos deben ser considerados como oportunidades de mejora por cuanto las actividades claves deben desarrollarse con la finalidad de asegurar que los procesos de la organización se planifiquen y desarrollen contemplando los requisitos logísticos del cliente. 
Tabla 3. Actividades claves

\begin{tabular}{ccccc}
\hline ITEMS & Promedio & Categoría & Promedio & Categoría \\
\hline $\begin{array}{c}\text { Planificación logística } \\
\text { Servicio al cliente }\end{array}$ & 83.33 & Alta presencia & & \\
Transporte & 54.16 & Moderada presencia & & Baja \\
Manejo de inventario & 12.5 & Muja presencia & $\mathbf{4 6 . 2 5}$ & presencia \\
$\begin{array}{c}\text { Flujo de información de } \\
\text { pedido }\end{array}$ & 45.83 & Baja presencia & & \\
\hline
\end{tabular}

Dicho resultado refleja que existen vacíos en la gestión logística en las unidades de compras analizadas, tal como lo expresa Mora (2006), razón por la cual es importante establecer indicadores de gestión logística para evitar barreras a la alta gerencia, en la identificación de los principales problemas presentes en la cadena logística, que perjudican ostensiblemente la competitividades de las instituciones universitarias públicas de la Costa Oriental del Lago.

Seguidamente, la tabla 4 muestra el resumen para la dimensión actividades de apoyo, reflejando de acuerdo a los encuestados, moderada presencia
(68,61\%) en aquellos procesos que proporcionan las personas y los recursos físicos como complemento a las actividades claves de la gestión logística en la unidad de compra de las instituciones universitarias públicas de la Costa Oriental del Lago, indicado por una concentración del 68,6\% en la opción afirmativa.

Las afirmaciones precedentes no alcanzan a validar los postulados de Ogalla (2005), quien señala que en estos procesos de apoyo o soporte, identifican aquellas actividades, que si bien, no son parte de la actividad principal de la organización, son necesarias e imprescindibles para la buena gestión y desarrollo de la misma.

Tabla 4. Actividades de apoyo

\begin{tabular}{|c|c|c|c|c|}
\hline ITEMS & Promedio & Categoría & Promedio & Categoría \\
\hline Almacenamiento & 66.67 & Moderada presencia & & \\
\hline $\begin{array}{l}\text { Manejo de } \\
\text { materiales }\end{array}$ & 50 & Moderada presencia & & \\
\hline Compras & 76.39 & Alta presencia & 68.61 & $\begin{array}{l}\text { Moderada } \\
\text { presencia }\end{array}$ \\
\hline $\begin{array}{l}\text { Cooperación con } \\
\text { producción }\end{array}$ & 100 & Alta presencia & & \\
\hline $\begin{array}{l}\text { Mantenimiento de } \\
\text { información }\end{array}$ & 50 & Moderada presencia & & \\
\hline
\end{tabular}


En este sentido, se debe considerar desuma importancia definir entre todos los actores, cuales son las actividades que van a proveer al proceso logístico del personal y los recursos físicos necesarios; adicionalmente, se debe establecer unsoporte de información adecuado a las necesidades de las instituciones universitarias públicas de la Costa Oriental del Lago, que cumpla con los requerimientos básicos para la planificación y control del proceso logístico.

En este recorrido de los resultados, se puede apreciar en la tabla 5, el resumen para la dimensión elementos de la gestión logística, la cual muestra un valor de 67,19\% de respuestas afirmativas, reflejando, de acuerdo a la opinión de los encuestados, moderada aplicación de un conjunto de elementos que son aprovisionamiento, producción, distribución física y abastecimiento, de manera que en las unidades de compra analizadas a juicio de la investigadora existen diferencias entre el nivel de servicio ofrecido y el percibido, lo que es contraproducente para las instituciones universitarias públicas de la Costa Oriental del Lago.

Estos resultados de moderada presencia permiten visualizar poca coincidencia con la teoría manejada por Taboada (1999), quien plantea que si se toma como referencia el flujo material, que es la columna vertebral de la logística, pueden entonces identificarse los elementos del sistema de gestión de logística los cuales son aprovisionamiento, producción, distribución física y abastecimiento.

Tabla 5. Elementos de la gestión logística

\begin{tabular}{|c|c|c|c|c|}
\hline ITEMS & Promedio & Categoría & Promedio & Categoría \\
\hline Aprovisionamiento & 50 & Moderada aplicación & \multirow{4}{*}{67.19} & \multirow{4}{*}{$\begin{array}{l}\text { Moderada } \\
\text { aplicación }\end{array}$} \\
\hline Producción & 100 & $\begin{array}{c}\text { Alta } \\
\text { aplicación }\end{array}$ & & \\
\hline Distribución física & 64.59 & Moderada aplicación & & \\
\hline Abastecimiento & 54.16 & Moderada aplicación & & \\
\hline
\end{tabular}

Así las cosas, es importante mantener un monitoreo continuo, bajo estudios de percepción de los clientes internos y proveedores, a fin de evaluar las variaciones para realizar los ajustes correspondientes a objeto de responder a los cambios, para así, cumplir con el propósito final de la logística, el cual es satisfacer al cliente en el momento en que el material e insumo, llegue a sus manos de manera oportuna, en las cantidades exactas, con excelente calidad, para poder atender correctamente a los usuarios.

Finalmente, la variable gestión logística se observa en la tabla 6 , un promedio de $60,68 \%$ que representa un moderado cumplimiento de la gestión logística dentro de las instituciones analizadas según el baremo establecido, lo cual le permite a la investigadora deducir la aplicación de mejoras en cada una de las actividades que no cumplieron con el porcentaje establecido, 
para así lograr el éxito en las unidades de compra que permita administrar estratégicamente el flujo y almacenamiento eficiente de los materiales requeridos por las dependencias académicas administrativas de las instituciones universitarias públicas de la Costa Oriental del Lago.

Los resultados expuestos, evidencia la moderada presencia de un conjunto de elementos destinados a prever y proveer los recursos necesarios que posibiliten realizar las actividades principales y de apoyo en tiempo, forma y al costo más oportuno para dirigir todos los cambios tanto estructurales como culturales de las instituciones universitarias públicas de la Costa Oriental del Lago para incrementar la competitividad y rentabilidad.

Tabla 6. Gestión logística

\begin{tabular}{lcccc}
\hline \multicolumn{1}{c}{ ITEMS } & Promedio & Categoría & Promedio & Categoría \\
\hline Actividades claves & 46.25 & Baja presencia & & Moderad \\
Actividades de apoyo & 68.61 & $\begin{array}{l}\text { Moderada } \\
\text { presencia }\end{array}$ & $\mathbf{6 0 , 6 8}$ & $\begin{array}{l}\text { a gestión } \\
\text { logística }\end{array}$ \\
$\begin{array}{l}\text { Elementos de la gestión } \\
\text { logística }\end{array}$ & 67.19 & $\begin{array}{l}\text { Moderada } \\
\text { aplicación }\end{array}$ & & \\
\hline
\end{tabular}

Visto así, existe una parcial congruencia con lo expuesto por Schroeder (2004), quien describe la gestión logística como el medio por el cual se satisfacen las necesidades de los clientes a través de la materia prima y sus operaciones hasta los proveedores. Destaca además el autor, que la misión de la gestión logística es planificar y coordinar todas las actividades necesarias para conseguir los niveles deseados de servicio y calidad al menor costo posible.

\section{CONCLUSIONES}

Con relación a las actividades claves de la gestión logística presentes en la unidad de compra de las instituciones universitarias públicas de la Costa Oriental del Lago, los resultados alcanzados, permiten reconocer que el personal logístico de las unidades estudiadas, identifican con alta presencia la planificación logística, moderada presencia el servicio al cliente, con baja presencia el transporte así como el flujo de información de pedido y con muy baja presencia el manejo de inventario.

En relación a la planificación logística en las unidades de compra de las instituciones analizadas se tiene presente que la planificación es vital para establecer un adecuado sistema logístico que requiere de un apropiado plan de implementación y operaciones. El resto de los indicadores de la dimensión analizada en este primer objetivo presentaron un comportamiento diferente a lo establecido en los postulados teóricos analizados

Así las cosas, no se capacita el personal para la atención al usuario, como tampoco se conoce la disponibilidad de vehículo al momento de realizar las compras, el manejo de inventario carece de un programa de registro, y los formatos de información existente no son actualizados frecuentemente. 
En cuanto alas actividades de apoyo de la gestión logística presentes en la unidad de compra de las instituciones universitarias públicas de la Costa Oriental del Lago, según los resultados se evidencio una moderada presencia en aquellos procesos que proporcionan las personas y los recursos físicos como complemento a las actividades claves de la gestión logística.

Los resultados obtenidos, conllevan a señalar que el almacenamiento en las unidades de compras analizadas no cuentan con un sistema que determinen los criterios para la selección del material, mientras que en lo relativo al manejo de materiales no existe combinación de métodos, que permiten alcanzar los objetivos establecidos, y es poco el mantenimiento de información que apoya a las actividades claves. Ahora bien, cabe acotar que las compras de materiales se hacen en la cantidad requerida según el plan establecido entre las diferentes dependencias y la unidad de compra.

En relación a los elementos de la gestión logística presentes en la unidad de compra de las instituciones universitarias públicas de la Costa Oriental del Lago, los resultados obtenidos dan evidencia de una moderada presencia del conjunto de elementos como son el aprovisionamiento, producción, distribución física y abastecimiento, por tanto, se deben realizar actividades coordinadas para dirigirlos y controlarlos.

Se evidencia que no se cuenta con un sistema de abastecimiento disponible de todos los productos en la cantidad real, por lo tanto los materiales comprados no abastecen las necesidades de las dependencias en el tiempo requerido, a pesar que las compras se realizan en consideración a los requerimientos de las diferentes dependencias.

Finalmente se concluye una moderadapresencia de la gestión logística dentro de las instituciones analizadas según el baremo establecido, validando lo planteado al inicio de la presente investigación, cuando el diagnóstico inicial permitió evidenciar la falta de un sistema de logística eficiente y eficaz dentro de sus procesos, de manera que las instituciones universitarias públicas de la Costa Oriental del Lago en sus unidades de compra parcialmente satisfacen las necesidades de las dependencias académicas administrativas cuando estas requieren de materiales.

\section{REFERENCIAS}

Acevedo, J.; Urquiega, A. J.; Gómez, M. (2001). Gestión de la Cadena de Suministro. Pentice-hall International. La Habana

Anaya, J. (2000). Logística integral. La gestión operativa de la empresa. Editorial ESICMadrid

Asamblea Nacional de la República Bolivariana de Venezuela Gaceta oficial de Nro. 360.129 de fecha 25 de marzo de 2008 decreto 5.929

Asamblea Nacional de la República Bolivariana de Venezuela Gaceta oficial de Nro. 37.688 de fecha 13 de mayo de 2013 decreto 2.371

Asamblea Nacional de la República Bolivariana de Venezuela Gaceta oficial de Nro. 38.567 de fecha 20 de noviembre de 2006 decreto 4.998

Asamblea Nacional de la República Bolivariana de Venezuela Gaceta oficial de Nro. 5.781 de fecha 20 julio de 2005 decreto 3.776

Asamblea Nacional de la República Bolivariana de Venezuela Ley de Universidades y Ley contra la corrupción Gaceta oficial Nro. 5.637

Ballou, R. (2004). Logística. Administración de la Cadena de Suministro. Quinta Edición. Prentice Hall, Pearson. México

Cespón, C.; Auxiliadora, M. (2003). Administración de la cadena de suministro: manual para estudiantes de la especialidad de Ingeniería Industrial. Tegucigalpa. Ed. UNITECHonduras 
Council of Logistics Management (2001). Annual Conference, Oak Brook, IL: Council of Logistics Management. Houston Tx

Ferrer, O.; Hirt, G.; Ramos, L. y Flores, M. (2004). Introducción a los negocios en un mundo cambiante. 4a ed., Ed. Mc Graw Hill. México DF, México

Garrioz, C. (2005) "La logística empresarial y la administración de inventario en Observatorio de la Economía Latinoamericana", CECSA, grupo Patria Cultural. México D.F.

González, J. (2005). Prácticas productivas y de aprovisionamiento en la industria de automoción: La importancia de la posición en la cadena de valor, Economía Industrial, n. 358, pp. 121-130

Ivancevich, J.; Lorenzi, P.; Skinner, S. (2000). Gestión: calidad y competitividad. 1ra ed. Ed. Mc Graw Hill. Madrid, España

Koontz, H., Weihrich, H (2003) Elementos de la administración, enfoque internacional. Sexta edición. Mc Graw. Interamericana de México, S.A. México

Lamb C, Hair J y McDaniel C (2002). Marketing, International Thomson Editors S.A. Pie de Imprenta, México

López, C (2001). Gestión Logística: herramienta indispensable para la competitividad.Madrid, España

Mañueco, A. (2004). Trabajando con los procesos: Guía para la Gestión por procesos. Junta de Castilla y León.Madrid, España
Montero, J. (2000). La distribución comercial: opciones estratégicas. Segunda Edición. ESIC Editorial. España

Mora, A. (2006). Indicadores Logísticos. Webpicking.com, Universidad Nacional de Medellín. Colombia

Ogalla, F. (2005). Sistema de Gestión: Una guía práctica. Ediciones Díaz de Santos. Madrid, España

Pau i Cos y De Navascues, R. (2000). Manual de logística integral. Mc Graw-Hill International. Madrid

Pérez, J. (2009).Gestión por procesos.3ra edición. Madrid, España, ESIC Editorial.

Porter, M. (2001). Estrategia Competitiva. Vigésima Octava Reimpresión. CECSA, grupo Patria Cultural. México D.F.

Reyes, E. (2001). Introducción a la logística internacional. Disponible en http://comerciointernacional.com.mx/in cludes/comercio/240/Logistica\%20intern acional\%20102002.pdf. Consultado: $24 / 02 / 2020$

Schroeder, R. (2004). Administración de operaciones concepto y casos contemporáneos. Segunda edición. Mc Graw Hill México

Stock; G. y Kasarda, J. (1999). "Logistics, Strategy and Structure". Mc Graw - Hill International Editions, Singapore

Taboada, R. Carlos (1999). Organización Planificación de la Producción. Ediciones pueblo y educación. La Habana 